Conclusion There is a substantial cost associated with the treatment of recurrence after ER which has not been considered in studies evaluating the efficacy of EMR. Injudicious prior manipulation contributes to the heavy cost burden. ESD should be evaluated more closely as it results in fewer subsequent treatments for recurrence and the possibility of longer surveillance intervals.

\section{PTH-017 REGISTRAR-LEVEL TRAINING IN COLORECTAL ESD IN WESTERN PRACTICE: OUTCOMES OF INDEPENDENT TRAINEE PERFORMED ESD}

Andrew Emmanuel*, Shraddha Gulati, Nishmi Gunasingam, Sophie Williams, Margaret Burt, Bu Hayee, Amyn Haji. King's College Hospital NHS Foundtaion Trust, London, UK

\subsection{6/gutjnl-2019-BSGAbstracts.42}

Introduction There are no published reports on registrar training in colorectal Endoscopic Submucosal Dissection (ESD) in western practice. It is unclear if any such training takes place in western centres where uptake of colorectal ESD has been slow and training, where it exists, is confined to experienced endoscopists who have already completed specialist training. We report our experience of registrar training in ESD and report outcomes from the first 10 cases completed by a trainee unassisted by a trainer.

Methods A registrar's ESD training over a 12 month period was evaluated after sequential training in colonoscopy, magnification endoscopy and endoscopic mucosal resection. The trainee had no prior first-operator experience in ESD. Cases were assigned by the trainer as either 'trainee to perform part' or 'trainee to complete'. Procedures were designated 'trainee completed, unassisted' if completed without the trainer handling the endoscope. A standardised ESD technique was employed. Outcomes for trainee completed cases included ESD proficiency (resection speed in $\mathrm{cm}^{2} /$ hour), R0 resection and complications.

Results The trainee performed part or complete colorectal ESD for 22 cases over the study period of which 10 were completed en bloc unassisted by trainer (mean size $48 \mathrm{~mm}$, range $22 \mathrm{~mm}-110 \mathrm{~mm}$ ). 8 of 9 cases assigned 'trainee to complete' were successfully completed unassisted, and 2 cases assigned 'trainee to perform part' were completed unassisted. Lesions were located in the rectum $(n=8)$ and sigmoid $(n=2)$. 1 case was performed under general anaesthetic due to extreme size $(110 \mathrm{~mm})$ and patient tolerance. Mean proficiency for unassisted trainee completed cases was $12.7 \mathrm{~cm}^{2} /$ hour. There were no perforations and 1 case of post procedure bleeding managed with observation alone. R0 resection was achieved in 100\%, including a case of submucosal invasive cancer.

Conclusions Registrar level training in ESD in western centres is feasible and safe. With appropriate prior diagnostic and EMR training, progress in ESD training is rapid and good proficiency (speed of resection) is achieved even for initial unassisted cases whilst clinical and oncological safe outcomes are maintained.

\section{PTH-018 SHORT-TERM OUTCOMES OF A PROTOCOL OF ESD/ HYBRID-ESD AS THE PRIMARY RESECTION STRATEGY FOR RECTAL ADENOMAS}

Andrew Emmanuel*, Shraddha Gulati, Christo Lapa, Sophie Williams, Nishmi Gunasingam, Margaret Burt, Bu Hayee, Amyn Haji. King's College Hospital NHS Foundtaion Trust, London, UK

\subsection{6/gutjnl-2019-BSGAbstracts.43}

Introduction ESD is rarely practiced in western centres. Given its technical difficulty, many western experts believe indications for ESD are limited. However, histopathologic diagnostic and treatment uncertainty resulting in over- or under-treatment can have grave consequences in the rectum. As a result, our unit recently opted for the exclusive use of ESD/Hybrid ESD to resect all large rectal adenomas. We report short term outcomes using this protocol for 12 months.

Methods Endoscopic resection (ER) of large $(\geq 20 \mathrm{~mm})$ colorectal adenomas were analysed and outcomes compared after adoption of an exclusive ESD resection strategy for all rectal adenomas $\geq 20 \mathrm{~mm}$ for 12 months (Period 2) compared to earlier resections (Period 1) when resection strategy was based on lesion morphology, surface characteristics and ER experience.

Results ER was performed for 185 rectal adenomas (period 1 $\mathrm{n}=154$, Period $2 \mathrm{n}=31$ ) with a mean size of $63 \mathrm{~mm}$ (range 20-160 mm). ESD/Hybrid ESD was used for 97\% of ER in Period 2 versus 61\% in Period $1(\mathrm{p}<0.001)$. A trainee performed part or all of $52 \%$ of ERs in Period 2 versus $7 \%$ in Period $1 \quad(p<0.001)$. There were no differences between time periods in complications (OR $0.4,95 \%$ CI $0.1-3.6, \mathrm{p}=0.44$ ) post procedure bleeding (OR 1.0, 95\% CI 0.1-8.8, $\mathrm{p}=1$ ) or risk of stenosis (OR 1.7, 95\% CI 0.2-16.7, $\mathrm{p}=0.66$ ). Rates of submucosal invasive cancer were similar $(12.9 \%$ versus $7.8 \%$, $\mathrm{p}=0.36)$. There were no clinically significant perforations in either group.

Conclusions ESD/Hybrid ESD for all large rectal adenomas, even when incorporating ESD training, is feasible and safe with sufficient expertise and experience. Data for long term outcomes are desirable to evaluate potential benefits in oncological results, reduced recurrence and potential fewer additional procedures.

\section{PTH-019 EFFICACY OF FCSEMS FOR REFRACTORY CBD STONES AT A SINGLE CENTRE OVER THREE YEAR PERIOD}

Kohilan Gananandan*, Cameron Green, Simon Phillpotts, Yasser El-Sherif, Sudeep Tanwar. Whipps Cross University Hospital, Barts Health NHS Trust, London, UK

\subsection{6/gutjnl-2019-BSGAbstracts.44}

Introduction $10-20 \%$ of patients have 'complex' CBD stones whereby extraction by ERCP is unsuccessful despite additional techniques, due to stone impaction and/or stone size. Cholangioscopy with laser/electrohydraulic lithotripsy is efficacious in patients with refractory stones but is normally only available in tertiary centres. Herein, we report the effect of introducing an 
additional modality for the extraction of 'complex' stones; fully covered self-expanding metal stent (fcSEMS) insertion alongside the 'complex' stone (Hartery et al, 2017) over a 3 year period at a single centre.

Methods Prospective study whereby patients with failed stone extraction by conventional techniques (sphincterotomy, sphincteroplasty - Boston CRE Balloon 10-20 mm, lithotripsy Olympus LithoCrushV, plastic stenting - 2 double pigtail stents 7-10Fr) underwent temporary fcSEMS insertion (Boston Wallflex $10 \mathrm{~mm} \times 6 \mathrm{~cm}$ or $8 \mathrm{~cm}$ ) alongside the complex stone for at least 4 weeks. Follow up ERCP was arranged and the fcSEMS was removed and stone extraction reattempted. Stones were classified as Cotton Grade $2(<10 \mathrm{~mm})$ or $3(\geq 10 \mathrm{~mm})$ with both cannulation and extraction rates assessed as ITT and per protocol.

Results Over a 36 month period 311 ERCPs were performed on 271 patients with CBD stones (238 Cotton Grade 2, 81 Cotton Grade 3). Cannulation at 1st ERCP was 95.2\% (258/ 271). Stone clearance at 1 st ERCP was $82 \%$ overall (221/ 271); 86\% (179/209) for Cotton Grade 2 and 68\% (42/62) for Cotton Grade 3. Stone clearance at 1 st or 2 nd ERCP was 91\% overall (247/271); 94\% for Cotton Grade 2 (196/209) and $82 \%$ for Cotton Grade 3 (51/62). Using conventional techniques 24 patients did not achieve CBD clearance of which 13 did not have a repeat ERCP (non-attendance, frailty or death).

A fcSEMS was inserted in 11 patients (stone size 9-33 $\mathrm{mm}$, age range 59-87 years). In 10 patients CBD clearance was achieved after a single SEMS insertion. With one patient a second fcSEMS insertion was required for a particularly large stone $(35 \mathrm{~mm})$ which did not fully fragment after the first procedure. Pancreatitis, perforation and cholangitis were not experienced in any of the patients in which a fcSEMS was inserted. One patient with a fcSEMS was later diagnosed with an extrahepatic cholangiocarcimoma but this was after CBD clearance had been achieved.

Conclusions The introduction of fcSEMS in a unit with cannulation rates and stone clearance rates above those mandated by the BSGE working group have resulted in very high stone extraction rate $(95 \%$ by ITT and $100 \%$ by per protocol analysis). Only one adverse event was noted in this study which is felt unlikely to be related to fcSEMS insertion. We suggest larger studies should be conducted to further evaluate the efficacy and safety of FCSEMS for 'complex' stones together with a formal health economic analysis.

\section{PTH-020 OUTCOMES OF ENDOSCOPIC STENTING FOR MALIGNANT DUODENAL OBSTRUCTION - EXPERIENCE OF A SINGLE CENTRE SERVICE}

Priyan Odedra, Vishal Amin, George Goodchild*, Harry Martin, Gavin Johnson, Stephen Pereira, George Webster, Michael Chapman. UCLH, London, UK

\subsection{6/gutjnl-2019-BSGAbstracts.45}

Introduction Duodenal obstruction may occur as a complication of duodenal, locally advanced or metastatic cancer. The resulting gastric outlet obstruction may reduce quality of life and life expectancy and the physiological mileu of malnutrition may interrupt palliative chemotherapy. We aim to evaluate of the technical and clinical outcomes of patients undergoing duodenal stent placement for malignant duodenal obstruction.
Methods Retrospective study of all patients referred for endoscopic duodenal stent placement from Jan 2014 - Jan 2018. Data was collected from endoscopy procedure records and electronic patient records. All patients with intent for duodenal stenting were included. Technical success was defined by endoscopic or radiographic evidence of satisfactory stent position. Outcome measures included removal of enteral tube (NG/NJ), cessation of vomiting, recommencement of oral nutrition, initiation or resumption of chemotherapy, complications and overall survival.

Results 50 patients were referred for duodenal stenting $(\mathrm{F}=31$, median age 68 years). CT scans were available for all patients and all cases were reviewed within our specialist MDM. The level of the obstruction was D1 (26/50), D2 (17/50) and D3 (7/50). Aetiology of malignant obstruction was: pancreatic $(\mathrm{n}=15)$, gastric $(\mathrm{n}=7)$, ovarian $(\mathrm{n}=6)$, cholangiocarcinoma $(\mathrm{n}=5)$, duodenal $(\mathrm{n}=5)$, colorectal $(\mathrm{n}=3)$, breast $(\mathrm{n}=2)$ and hepatocellular $(n=1)$. Stenting was attempted in 48 patients (2 were deemed unfit). Successful stent placement was achieved in $43 / 48(90 \%)$ of patients, with a failure to stent the remaining 5 patients due to an inability to cross the stricture with a wire. A total of 51 uncovered, self-expanding $22 \mathrm{~mm}$ diameter metal stents were placed in 43 patients ( 1 patient required 2 stents at the index procedure, 6 patients required 1 further stent and 1 patient required 2 further stents,). The median stent length was $9 \mathrm{~cm}$ (range 6-12 cm). Technical success rates were $90 \%$. Clinical success rates were as follows: Subsequent removal of enteral tube post-procedure $68 \%$, cessation of vomiting $64 \%$, recommencement of oral diet $58 \%$, further chemotherapy 29\%. Overall 28 day survival was $85 \%$ and 6 month survival was $33 \%$ (median survival 66 days). There were no immediate complications following stent placement. 1 patient developed biliary sepsis 5 days post-stenting (previously normal LFTs) and died within 28 days. 3 patients developed obstructive jaundice following stenting (at 4 months, 8 months and 21 months), which was successfully treated with percutaneous stenting in all cases.

Conclusion Providing a guidewire can be passed across the stricture, duodenal stenting for malignancy has a high technical success rate in our centre. Early clinical benefit is seen in the majority of patients, and of the 41 patients alive at 3 months, only $3(7 \%)$ required re-intervention during this period. At 6 months, 16 patients were alive, 7 of whom $(44 \%)$ had required re-intervention. This single centre experience demonstrates high technical and clinical success rates for stenting in patients with malignant duodenal obstruction, with benefit maintained well beyond 3 months. In those patients that survived $>6$ months, half had further chemotherapy.

\section{PTH-021 PROXIMALLY MIGRATED PANCREATIC STENTS - ENDOSCOPIC MANAGEMENT AND OUTCOME AT A TERTIARY CENTRE}

${ }^{1}$ George Goodchild*, ${ }^{2} J o n a t h a n$ Potts, ${ }^{1}$ Harry Martin, ${ }^{2}$ Douglas Thorburn, ${ }^{1}$ Michael Chapman, 'Gavin Johnson, 'Stephen Pereira, 'George Webster. 'UCLH, London, UK; ${ }^{2}$ Royal Free Hospital, London, UK

\subsection{6/gutjnl-2019-BSGAbstracts.46}

Introduction Pancreatic stents (PS) are increasingly used to aid biliary cannulation and reduce the risk of pancreatitis during endoscopic retrograde cholangiopancreatography (ERCP). Proximal PS migration is uncommon (reported in 2-5\%) but poses a significant problem due to the risk of pancreatitis and pain. 